\title{
Somatic Growth of Children with Biliary Atresia After Living Related Liver Transplantation
}

\author{
Hironori Kato, Takashi Nishizawa, Shinji Uemoto, Kaoru Sano, Isao Shirahase, \\ Eiji Yamamoto, Koichi Tanaka, Kazue Ozawa, Chutaro Yamanaka and Haruki Mikawa
}

Second Department of Surgery and Department of Pediatrics, Faculty of Medicine, Kyoto University, Kyoto, Japan

\begin{abstract}
We measured height and serum levels of IGF-1/somatomedin C in 15 patients before and after liver transplantation. They all had liver cirrhosis due to biliary atresia and received an orthotopic partial liver transplant from a living parent (ages eight months to nine years, mean 3.5 years.). Their follow-up period after liver transplant was from five to 22 months (mean 13 months). Their immunosuppressive therapy was mainly FK506 and steroids. We define improvement of somatic growth as increase of standard deviation (SD) score in height at the last follow-up as compared with preoperative SD. The liver transplant patients were divided into two groups (group A, 7 patients whose preoperative height $>-1.5 \mathrm{SD}$; group B, eight patients whose preoperative height $<-1.5 \mathrm{SD}$ ). Six in group A and two in group B showed improvement of growth. Plasma level of IGF-1 increased in all patients in group A and six in group B within three months after liver transplantation. These results suggest that liver transplantation can improve the somatic growth of children with biliary atresia within one year when it is performed before the onset of severe growth retardation.
\end{abstract}

Key words: liver transplantation, somatic growth, IGF-1/somatomedin C, FK506

\section{Introduction}

Most children with biliary atresia who progress to end-stage liver disease in spite of portoenterostomy are candidates for liver transplantation. They show growth retardation due to deficient protein synthesis, fat malabsorption, hepatic rickets, severe infec-

Correspondence: Hironori Kato, M.D., Second Department of Surgery, Faculty of Medicine, Kyoto University, 54, Kawara-cho, Shogoin, Sakyo-ku, Kyoto 606 Japan tion, etc. [1]. One of the aims of pediatric liver transplantation is to alleviate growth retardation and improve the quality of life. Therefore, one of the key points of posttransplant management is to maintain normal growth.

Growth after liver transplantation has been reported to be normal or catch-up with cyclosporine and prednisolone. In this study we report somatic growth and plasma level of IGF- 1 in 15 patients with biliary atresia before and after living related liver transplantation and treatment with FK506. 


\section{Patients and Methods}

Between June 1990 and September 1991, 15 liver transplantations were performed on 15 patients with biliary atresia at our institution. They all received an orthotopic partial liver transplant from a living parent (six male, nine female; ages eight months to nine years, mean 3.5 years.). They all survived for more than five months after transplantation. Two patients died during the first six months, one of aspiration asphyxia and one of sepsis. Their follow-up after liver transplant was from five months to 22 months (mean 13 months). Their immunosuppressive therapy was mainly FK506 and steroids. Steroids were discontinued after several months (from one to 12 months, mean 4.6 months). Thirteen patients who are alive at present are being treated with FK506 alone.

Height was measured every two to three months and plotted on the Japanese national growth standard in 1988 . We define improvement of growth as increase of standard deviation (SD) score in height at the last follow-up as compared with preoperative SD score.

The liver transplant patients were divided into two groups. Group A consists of seven patients whose growth was normal (preoperative height $>-1.5 \mathrm{SD}$ ) (five male, two female; ages 10 months to seven years, mean 3.6 years.). Group B consists of eight patients who showed growth retardation (preoperative height $<-1.5 \mathrm{SD}$ ) (one male, seven female; ages eight months to nine years, mean 3.3 years.).

Blood samples were also drawn one and three months after transplantation. Plasma levels of IGF-1 were directly determined by RIA kit.

\section{Results}

In height, six in group $\mathrm{A}$ and two in group B showed improvement of growth (Table 1). Standard deviation scores of height one
Table 1. Improvement (pre < post) ratio in standard deviation score (SDS) of height and IGF-1 level after liver transplantation

\begin{tabular}{ccl}
\hline group & SDS of height & IGF-1 level \\
\hline A & $6 / 7(86 \%)$ & $7 / 7(100 \%)$ \\
B & $2 / 8(25 \%)$ & $6 / 8(75 \%)$ \\
\hline
\end{tabular}

Table 2. Standard deviation score of height before and after transplantation

\begin{tabular}{cll}
\hline group & Before transplant & After transplant \\
\hline A & $-0.406 \pm 0.930$ & $-0.067 \pm 0.716$ \\
& $(-1.29-1.15)$ & $(-0.89-1.44)$ \\
B & $-3.111 \pm 1.613$ & $-3.150 \pm 2.039$ \\
& $(-6.10--1.66)$ & $(-6.80--0.15)$ \\
\hline
\end{tabular}

Values are mean \pm SD, with range in parentheses.

Table 3. Plasma level of IGF-1 (U/ml) before and after transplantation

\begin{tabular}{cccc}
\hline group & Before transplant & \multicolumn{2}{c}{ After transplant } \\
& 1 month & 1 month & 3 months \\
\hline A & $0.281 \pm 0.125$ & $1.832 \pm 1.522$ & $2.454 \pm 1.508$ \\
& $(0.17-0.52)$ & $(0.40-4.24)$ & $(0.43-3.88)$ \\
B & $0.247 \pm 0.136$ & $2.185 \pm 2.720$ & $1.947 \pm 1.771$ \\
& $(0.15-0.57)$ & $(0.33-6.56)$ & $(0.17-4.56)$ \\
\hline
\end{tabular}

Values are mean $\pm \mathrm{SD}$, with range in parentheses.

month before transplant and at the last follow-up are shown for groups A and B (Table 2).

Plasma level of IGF-1 increased in all seven patients in group $\mathrm{A}$ and in six in group $\mathrm{B}$ three months after transplant as compared with the preoperative level (Table 1). Plasma levels of IGF-1 one month before, one month after and three months after transplantation are shown for groups A and B (Table 3).

\section{Discussion}

Although various complications occurred in our patients with biliary atresia, especially in those whose liver function was severely impaired, the overall results were satisfactory. No patients or grafts were lost during the first five months after transplantation.

Growth rate has been reported to decrease during the first year after liver transplantation 
[2-4]. The decrease has been correlated with the dose of prednisolone [3]. Growth after the first year has generally been good [2]. On the other hand, accelerated growth immediately after liver transplantation has also been reported in patients receiving cyclosporine and prednisolone [5].

In our patients with normal pre-operative growth, relative height improved during the first six to 12 months. The discontinuation of steroids may have contributed to this improvement. In the patients with pre-operative growth retardation, relative height was slightly reduced during the first year. This is probably due to the prolonged convalescence period and many complications after liver transplantation.

Preoperative IGF-1 level was low in most of our patients, even in those with normal growth. This indicates that a cirrhotic liver with biliary atresia excretes IGF-1 poorly in response to endogenous growth hormone, and a low level of IGF-1 is one of the causative factors for growth retardation. In most patients, liver graft excreted IGF-1 well immediately after liver transplantation. Although the growth improvement of patients after liver transplantation is multifactorial, it is induced to some extent by an increase in IGF-1.
Our results suggest that liver transplantation can improve the somatic growth of children with biliary atresia within one year if it is performed before the onset of severe growth retardation.

\section{References}

1. Burgess DB, Martin HP, and Lilly JR. The development status of children undergoing the Kasai procedure for biliary atresia. Pediatrics 1982; 70: 624-9.

2. Urbach AH, Gartner JC, Malatack JJ, et $a l$. Linear growth following pediatric liver transplantation. AJDC 1987; 141: 547-9.

3. Stewart SM, Uauy R, Waller DA, et al. Mental and motor development, social competence, and growth one year after successful pediatric liver transplantation. J Pediatr 1989; 114: 574-81.

4. Laine J, Holmberg C, Sipilä I, et al. Growth and renal function after liver transplantation in children. Transplant Proc 1992; 24: 398-400.

5. Sopolidoro JVN, Berquist WE, Pehlivanoglu E, et al. Growth acceleration in children after orthotopic liver transplantation. J Pediatr 1988; 112: 41-4. 